\title{
Ifosfamide with Regional Hyperthermia in Soft-Tissue Sarcomas
}

\author{
M. Schlemmer ${ }^{a}$ C.M. Wendtner ${ }^{a}$ R.D. Issels ${ }^{a, b}$ \\ a Department of Internal Medicine III, Klinikum Grosshadern Medical Center, Ludwig Maximilians University \\ Munich, and ${ }^{b}$ Clinical Cooperation Group on Hyperthermia, GSF - National Research Center for Environment and \\ Health, Institute of Molecular Immunology, Munich, Germany
}

\section{Key Words}

Soft tissue sarcoma $\cdot$ Ifosfamide $\cdot$ Regional

hyperthermia $\cdot$ Neoadjuvant chemotherapy

\begin{abstract}
For high-risk soft tissue sarcomas (HR-STS) of adults, new treatment strategies are needed to improve outcome with regard to local control and overall survival. Therefore, systemic chemotherapy has been integrated either after (adjuvant) or before (neoadjuvant) optimal local treatment by surgery and radiotherapy in HR-STS. The combination with regional hyperthermia as a new treatment strategy seems to open a new therapeutic window.
\end{abstract}

Copyright @ 2003 S. Karger AG, Basel

\section{High-Risk Soft Tissue Sarcomas}

Soft tissue sarcomas (STS) represent a heterogeneous group of relatively rare malignancies with a wide spectrum in terms of histologic type and prognosis [1]. In the last decade the use of limb-sparing surgery and postoperative irradiation has become standard practice in the management of resectable extremity STS with 5-year rates of local control similar to those reported with radical resection or amputation [2, 3]. However, the anatomic location (e.g. retroperitoneal, visceral, RP/V) and invasiveness of
STS often prevent resection with adequate margins [4], and the toxicity of radiotherapy limits the use of potentially therapeutic doses with negative impact on local control [5]. In addition, unfavorable prognosis of STS is determined by high-grade histology, large tumor size ( $\geq 5 \mathrm{~cm}$ ) and deep localization [6]. While median overall survival (OS) for patients with extremity lesions is 33 months, patients with high-grade tumors of the retroperitoneum were reported to have an even worse prognosis with a median OS between 9 and 20 months despite surgical resection [7]. Recently it was shown that RP/V site is an additional poor prognosticator independent of other factors like tumor size, grade and recurrence [8]. Typically the majority of patients with RP/V tumors frequently die with local disease without evidence of distant metastasis at the time of death. Further adjuvant treatment modalities like early postoperative intraperitoneal Adriamycin, high-dose adjuvant radiotherapy with protecting silicone implants or IORT were explored in small patient series.

As a reflection of these obstacles, the strategy of systemic chemotherapy has been integrated in the multidisciplinary approach either after (adjuvant) or before and after (neoadjuvant) locally curative treatment, mainly in order to eradicate distant micrometastases thought to be present at the time of diagnosis. Once inoperative metastatic disease with progression becomes measurable, the intention of systemic chemotherapy remains only palliative.

\section{KARGER \\ Fax + 41613061234 \\ E-Mail karger@karger.ch \\ www.karger.com

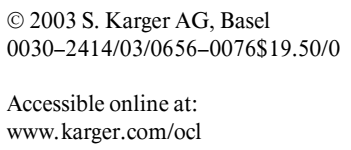

Dr. R.D. Issels

Department of Internal Medicine III, Klinikum Grosshadern Medical Center Ludwig Maximilians University Munich

DE- 81377 Munich (Germany)

E-Mail issels@med3.med.uni-muenchen.de 


\section{Rationale of Regional Hyperthermia Combined with Systemic Chemotherapy}

Within the last 15 years, regional hyperthermia (RHT) as a new treatment modality has entered clinical trials [9]. The results of phase II studies in pretreated and locally advanced patients with STS are encouraging and justify further testing in randomized studies as first-line treatment for well-defined risk groups [10].

The rationale for the combination of cytotoxic drugs with hyperthermia in the treatment of high-risk STS (HRSTS) is based on experimental evidence that heat exposure increases the killing of tumor cells by direct thermal cytotoxicity and is able to sensitize perfused tumor tissue towards chemotherapy in a synergistic manner [11]. In addition, recent results indicate that heat-shock proteins induced in tumor cells under hyperthermic stress are able to elicit specific T- and NK-cell immune responses [12]. Hyperthermia in combination with radiotherapy as neoadjuvant treatment for HR-STS patients has already shown to impact positively on local tumor control, whereas it did not seem to influence the rate of distant metastases or survival [13].

In an effort to improve both local tumor control and micrometastases, a multimodality treatment strategy including RHT combined with chemotherapy seemed attractive to be explored in HR-STS. The objective of this report is to determine response rate and survival parameters for patients with HR-STS at different sites after neoadjuvant thermochemotherapy.

\section{Neoadjuvant Chemotherapy Alone}

Preoperative chemotherapy (chemotherapy delivered before local therapy) has some advantages over postoperative treatment, because the presence of evaluable tumor allows the determination of chemotherapy sensitivity clinically as well as pathologically, and valuable prognostic information may be obtained. The primary tumor may also be downstaged, and some lesions that were technically unresectable may become more amenable to surgical intervention. In addition, drug delivery to the primary tumor may be improved because surgery and radiation therapy have not yet been delivered and the tumor's vascular supply remains undisturbed.

The EORTC has completed the first randomized trial on neoadjuvant chemotherapy in patients with resectable STS of the extremities, head, neck and trunk. Only highrisk patients were eligible, based on tumor size $(>8 \mathrm{~cm})$ or intermediate/high histological grade (mitotic count $\geq 3$ / 10 high-power fields). Three courses of chemotherapy (doxorubicin $50 \mathrm{mg} / \mathrm{m}^{2}+$ ifosfamide $5 \mathrm{~g} / \mathrm{m}^{2}$ ) were administered before definitive surgery and radiotherapy. Chemotherapy had to start within 6 weeks of biopsy or at an attempt at definitive surgery. Patients in the control arm proceeded to immediate surgery and postoperative radiotherapy. 150 patients were entered into the study and 134 were eligible, 67 in each arm [14]. This trial was closed after completion of phase II since accrual was insufficient to justify expanding the study into the scheduled phase III study. Objective response rate to preoperative chemotherapy in 49 patients assessable for response was $29 \%$. At a median follow-up of 7.3 years, the 5-year DFS was 52\% (control arm) and 56\% (chemotherapy arm), respectively $(\mathrm{p}=0.35)$.

Several phase II studies of neoadjuvant chemotherapy have been carried out in patients with bulky STS according to risk factors (tumor size, grade, inadequate surgery, local recurrence). It appeared that some patients benefit from chemotherapy because formerly inoperable cases were rendered operable [15-17].

\section{Clinical Results of RHT Combined with Neoadjuvant Chemotherapy}

In 1986, at the Klinikum Grosshadern Medical Center (KGMC), we initiated a phase II study for patients with advanced chemoresistant STS combining second-line chemotherapy with RHT [18]. These patients had relapsed after prior surgery and radiation, and had not responded to previously given doxorubicin-based chemotherapy alone. A drug combination of ifosfamide + etoposide (VP-16) was used, combined with RHT as a secondline treatment. The local response rate was $37 \%$. Besides long-term tumor control in a subgroup of patients, the analysis of tumor temperatures (e.g. $\mathrm{T}_{20}, \mathrm{~T}_{50}, \mathrm{~T}_{90}$ ) achieved using an electromagnetic deep regional-heating device (BSD system) showed significantly higher temperature parameters in responders than in non-responders. The results have been confirmed in the extended trial including 65 patients with chemo-pretreated sarcomas [19].

Thereafter, two additional consecutive phase II studies at the KGMC were performed in HR-STS but these patients had not received prior chemotherapy (chemonaive patients). In the first study (RHT-91 protocol), 59 patients received 4 cycles of EIA consisting of etoposide, ifosfamide and doxorubicin combined with concurrent 
RHT. The RHT was applied using the BSD system and the aim was to achieve a maximum tumor temperature of $\geq 42^{\circ} \mathrm{C}$ for a period of $60 \mathrm{~min}$. Following this treatment, those patients whose tumor was judged to be resectable underwent surgery. If the tumor showed signs of response, 4 additional cycles of combined chemotherapy and hyperthermia were given. All patients not preirradiated also received external beam radiotherapy. Treatment-related toxicity was acceptable. Median survival was 52 months and the 5-year survival rate was 49\% (95\% CI 36-61\%). The data are in favor of the patients responding to EIA combined with hyperthermia [20].

In the second study (RHT-95 protocol), a similar highrisk patient group (54 patients) received the same treatment as in the RHT-91 protocol with the exception that the patients did not receive hyperthermia but chemotherapy alone after surgery. This study showed an inferior local failure-free survival (LFFS) rate compared with the RHT-91 protocol, but no difference in overall survival [21]. Thus, postsurgical hyperthermia may be critical for local control.

From 1991 to 1997, a total of 58 patients with HR-STS at RP/V sites were prospectively treated with 4 cycles of etoposide, ifosfamide and doxorubicin combined with RHT followed by surgery, adjuvant chemotherapy and radiation. Objective response rate assessable in 40 patients was $13 \%$ (5 partial responses). Including minor responses $(n=8)$, the radiographic response rate was $33 \%$. Pathologic response rate assessable in 26 patients after surgical resection was $42 \%$. Median OS was 31 months. At a median observation time of 74 months, 5-year probability of LFFS, distant metastasis-free survival, event-free survival and OS were 25, 51, 20 and 32\%, respectively. Averaged minimum temperatuers $\left(\mathrm{T}_{\min }\right)$ and time-averaged temperatures achieved in $50 \%\left(\mathrm{~T}_{50}\right)$ and $90 \%\left(\mathrm{~T}_{90}\right)$ of all measured tumor sites differed significantly between responders and non-responders $\left(\mathrm{T}_{\min }: 39.3\right.$ vs. $38.0^{\circ} \mathrm{C}$, $\mathrm{p}=0.002 ; \mathrm{T}_{50}: 40.9$ vs. $40.3^{\circ} \mathrm{C}, \mathrm{p}=0.038 ; \mathrm{T}_{90}: 40.1 \mathrm{vs}$. $\left.39.3^{\circ} \mathrm{C}, \mathrm{p}=0.017\right)$. At 5 -year follow-up, probability of LFFS (59 vs. $0 \%$; p < 0.001$)$ and OS (60 vs. $10 \%$; p < 0.001 ) was significantly in favor of patients responding to neoadjuvant thermochemotherapy [22]. It is important to note that besides inclusion of unfavorable anatomic tumor sites and recurrent STS, the study protocol allowed inclusion of patients who had tumors that were not adequately resectable before entering the study. This argues for a high burden of patients with unfavorable prognosis in our study in contrast to other neoadjuvant treatment protocols where only patients were included who were judged to have resectable tumors before the start of neoadjuvant chemotherapy [15]. The fact that $\mathrm{R} 0$ resection was achieved in a proportion of patients after neoadjuvant treatment supports the idea of a tumor downstaging. Moreover, it was demonstrated that previously resected patients who had only microscopic tumor left at the start of protocol treatment had a significantly higher probability of local relapse in comparison with patients whose tumor was resected after neoadjuvant thermochemotherapy.

The EORTC Soft Tissue and Bone Sarcoma Group (STBSG) is further testing this multimodal concept as a first-line treatment of HR-STS in adults in a multicenter prospective phase III trial (EORTC 62961/ESHO RHT95), as an Intergroup study with the European Society of Hyperthermic Oncology (ESHO) [23]. Patients meeting all of the eligibility criteria at first presentation (tumor size $\geq 5 \mathrm{~cm}$, grade II or grade III, extracompartmental) or after inadequate surgery (resections with microscopic/ macroscopic residual tumor) are entered in this protocol with the intention of improving local tumor control and early prevention of systemic metastasis. According to the inclusion criteria defined above, the majority of patients with HR-STS recruited for this multimodality treatment protocol cannot be controlled by standard procedures. All eligible patients will be randomized to receive either 4 cycles of EIA (etoposide $250 \mathrm{mg} / \mathrm{m}^{2}+$ ifosfamide $6 \mathrm{~g} / \mathrm{m}^{2}+$ doxorubicin $50 \mathrm{mg} / \mathrm{m}^{2}$ ) within 12 weeks (chemotherapy arm) or the same EIA regimen combined with RHT (RHT arm). The patients will then receive optimal local treatment using adequate surgery immediately followed by radiotherapy. After the completion of radiotherapy an additional 4 cycles of EIA chemotherapy alone or in combination with RHT will be given. The application of RHT with external annular phased array applicators (60$100 \mathrm{MHz}$ ) results in a selective temperature elevation (range $40-44^{\circ} \mathrm{C}$ ) within the tumor and the directly adjacent tissue. The EIA regimen of the neoadjuvant protocol combines conventional doses of the most active single agents (doxorubicin + ifosfamide) in STS with etoposide being added prior to RHT on day 1 and 4 of each cycle. This combination of systemic EIA chemotherapy and RHT has been shown to be effective in HR-STS and allows an adequate conservative surgical approach after 4 EIA/RHT cycles in responding patients for whom radical resection without mutilation (e.g. hemipelvectomy, amputation) was formerly considered impossible [20].

At present more than 180 patients have been recruited for this Intergroup study. For the final analysis, about 170 patients are required in each treatment arm. 


\section{Conclusions}

A short description of the background, the special design, and the rationale of phase II and an ongoing phase III protocol for patients with HR-STS is given including RHT combined with systemic chemotherapy. Clinical advances in the treatment of STS are often hampered by the difficulty in accrual of patients for prospective clinical trials. The intention of this review is to emphasize that collaboration among European institutions and among the individual oncologist is the only basis that can provide the answer for the definitive role of new strategies such as RHT in the treatment of HR-STS.

\section{References}

1 Enzinger FM, Weiss SW: Soft Tissue Tumors. St Louis, Mosby, 1988.

2 Pisters PWT, Harrison LB, Leung DHY et al: Long-term results of a prospective randomized trial of adjuvant brachytherapy in soft tissue sarcoma. J Clin Oncol 1996;14:859-868.

3 Yang JC, Chang AE, Baker AR et al: Randomized prospective study of the benefit of adjuvant radiation therapy in the treatment of soft tissue sarcomas of the extremity. J Clin Oncol 1998;16:197-203

4 Alvarenga JC, Ball ABS, Fisher C et al: Limitations of surgery in the treatment of sarcoma. $\mathrm{Br}$ J Surg 1991;78:912-916.

5 Fein DA, Lee WR, Lanciano RM et al: Management of extremity soft tissue sarcomas with limb-sparing surgery and postoperative irradiation: Do total dose, overall treatment time and the surgery-radiotherapy interval impact on local control? Int J Radiat Oncol Biol Phys 1995 32:969-976.

6 Gaynor JJ, Tan CC, Casper ES et al: Refinement of clinicopathologic staging for localized soft tissue sarcoma of the extremity: A study of 423 adults. J Clin Oncol 1992;10:1317-1327.

7 Bautista N, Su W, O’Connell TX: Retroperitoneal soft-tissue sarcomas: Prognosis and treatment of primary and recurrent disease. Am Surg 2000;66:832-836.

8 Makela J, Kiviniemi H, Laitinen S: Prognostic factors predicting survival in the treatment of retroperitoneal sarcoma. Eur J Surg Oncol 2000;26:552-555.

9 Falk M, Issels RD: Hyperthermia in oncology. Int J Hyperthermia 2000;17:1-16.
10 Nielsen OS, Horsman M, Overgaard J: A future for hyperthermia in cancer treatment? Eur J Cancer 2001;37:1587-1589.

11 Dewey WC: Interaction of heat with radiation and chemotherapy. Cancer Res 1984;44:47144720.

12 Hyperthermia and the immune systeme (special issue). Int J Hyperthermia 2002;18:486608.

13 Prosnitz LR, Maguire P, Anderson JM et al: The treatment of high-grade soft tissue sarcomas with preoperative thermoradiotherapy. Int J Radiat Oncol Biol Phys 1999;45:941-949.

14 Gortzak E, Azzarelli A, Buesa J et al: A randomised phase II study on neoadjuvant chemotherapy for 'high-risk' adult soft-tissue sarcoma. Eur J Cancer 2001;37:1096-1103.

15 Pezzi CM, Pollock RE, Evans HL et al: Preoperative chemotherapy for soft-tissue sarcomas of the extremities. Ann Surg 1999;211:476481.

16 Rouesse JG, Friedman S, Sevin DM et al: Preoperative induction chemotherapy in the treatment of locally advanced soft tissue sarcomas. Cancer 1987;60:296-300.

17 Pisters PWT, Patel SR, Varma DGK et al: Preoperative chemotherapy for stage IIIB extremity soft tissue sarcoma: Long-term results form a single institution. J Clin Oncol 1997;15:34813487.

18 Issels RD, Prenninger SW, Nagele A et al: Ifosfamide plus etoposide combined with regional hyperthermia in patients with locally advanced sarcomas: A phase II study. J Clin Oncol 1990; 8:1818-1829.
19 Issels RD, Mittermüller J, Gerl A et al: Improvement of local control by regional hyperthermia combined with systemic chemotherapy (ifosfamide plus etoposide) in advanced sarcomas: Updated report on 65 patients. J Cancer Res Clin Oncol 1991;117(suppl 4):141147.

20 Issels RD, Abdel-Rahman S, Wendtner CM et al: Neoadjuvant chemotherapy combined with regional hyperthermia for locally advanced primary or recurrent high-risk soft tissue sarcomas of adults: Long-term results of a phase II study. Eur J Cancer 2001;37:1599-1608.

21 Wendtner CM, Abdel-Rahman S, Baumert J et al: Treatment of primary, recurrent or inadequately resected high-risk soft tissue sarcomas of adults: Results of a phase II pilot study (RHT-95) of neoadjuvant chemotherapy combined with regional hyperthermia. Eur J Cancer 2001;37:1609-1616.

22 Wendtner CM, Abdel-Rahman S, Krych M et al: Response to neoadjuvant chemotherapy combined with regional hyperthermia predicts long-term survival for adult patients with retroperitoneal and visceral high-risk soft tissue sarcomas. J Clin Oncol 2000;20:3156-3164.

23 EORTC 62961/ESHO RHT-95: Randomized study comparing neoadjuvant chemotherapy etoposide + ifosfamide + adriamycin (EIA) combined with regional hyperthermia versus neoadjuvant chemotherapy alone in the treatment of high-risk soft tissue sarcomas in adults http://www.med.uni-muenchen.de/tzm/eortc 